УДК 51.75

DOI $10.21661 / \mathrm{r}-555460$

\title{
A.В. Танюхин
}

\section{ФАКТОРНАЯ МОДЕЛЬ ОЦЕНКИ БУДУЩИХ ПРИРАЩЕНИЙ УБЫТКОВ ОТ СОБЫТИЙ ПРОШЛЫХ ПЕРИОДОВ В ЦЕЛЯХ БОЛЕЕ ТОЧНОЙ АКТУАРНОЙ ТАРИФИКАЦИИ}

Аннотация: в данной статье рассмотрены проблемы применения многофакторных моделей в целях актуарной тарификаиии. Отмечены недостатки существующих методов оченки резервов убытков (IBNR) с точки зрения последующего применения многофакторных моделей для исчисления тарифа. Предложен подход к резервированию убытков в иеелях актуарной тарификаџии на основе обобщенных линейных моделей. Данный подход позволяет более точно учитывать влияние отдельных тарифных факторов на прочессы приращения убытков в будущем от событий прошлых периодов.

Ключевые слова: актуарные расчеты, резерв убытков, поздние убытки, нормированные приращения убытков, обобщенная линейная модель, перекрестная параметризащия, нетто-тариф.

\section{Введение}

В настоящее время в практике страхования, не связанного со страхованием жизни, широко используются постулаты теории риска в целях исчисления неттотарифа. В соответствии с данной теорией верным размером нетто-тарифа при риско-нейтральном подходе является математическое ожидание убытка по риску $[1$, c. 28]. Теория риска исходит из возможности наличия множества однородных рисков в страховом портфеле. Таким образом становится возможной статистическая оценка данного математического ожидания (нетто-тарифа). Однако, существуют некоторые практические проблемы применения теории. Так, например, наличие договоров страхования с одинаковыми сроками еще не означает возможности учета убытков по ним, как наблюденных значений одной и той же случайной величины, договоры страхования, как правило, начинаются в разные 
даты, что создает различия в вероятностях наступления страховых событий по ним на дату оценки (один договор мог действовать, например, девять месяцев в рассматриваемом актуарием году, другой - всего лишь один месяц). Также известна проблема поздно учитываемых убытков по событиям рассматриваемого периода, имеющая на текущий момент определенное актуарное решение. Об этой проблеме и существующих недостатках такого решения пойдет речь в данной работе.

В части первой производится обзор наиболее популярных моделей оценки поздно учитываемых убытков и ставится проблема практического дальнейшего применения при условии тарификации с применением многофакторных моделей.

В части второй предлагается решение данной проблемы.

Часть I. Модели оценки поздно учитывваемых убытков (IBNR)

Модели оценки IBNR детально и подробно рассмотрены в литературе. Так, например, Т. Мак приводит достаточно подробный обзор существующих наиболее популярных моделей. Можно отметить основные:

- модель независимых нормированных приращений убытков (МНП) [2, c. 205];

- модель Борнхьютера-Фергюсона (МБФ) [2, с.215];

- модель цепной лестницы (МЦЛ) [2, с.216];

- модель Бюльмана-Штрауба (МБШ) [2, с.208];

- модели на основе перекрестной параметризации нормированных приращений убытков (МПП) [2, с.239].

Модели МНП и МБФ похожи «усреднением» оценок математических ожиданий размера поздно учитываемых убытков по периодам событий. Модели МЦЛ, МБШ, МПП тем или иным образом проводят различия между периодами событий. Эти модели позволяют получить поправочные коэффициенты за каждый период события. Кроме того, в предыдущей работе [3] показано, что МЦЛ 
может быть представлена как частный случай МПП, а именно обобщенной линейной модели перекрестной параметризации нормированных приращений убытков, имеющих распределение Пуассона.

Недостаток всех этих подходов в современных условиях следующий.

В большинстве случаев обеспечение конкурентоспособности на рынке страхования требует построения многофакторных моделей с целью более детальной тарификации рисков. На практике для построения многофакторной модели вначале получаем таблицу вида табл.1.

Таблица 1

Датасет для построения многофакторной модели нетто-тарифа

\begin{tabular}{|c|c|c|c|c|c|}
\hline Фактор 1 & Фактор 2 & $\ldots$ & Фактор 1 & $\begin{array}{l}\text { Экспозиция } \\
\text { риска }\end{array}$ & $\begin{array}{l}\text { Средний } \\
\text { убыток } \\
\text { (частота) }\end{array}$ \\
\hline $\begin{array}{c}\text { Уровень 1 } \\
\text { Фактора 1 }\end{array}$ & $\begin{array}{c}\text { Уровень 1 } \\
\text { Фактора 2 }\end{array}$ & $\ldots$ & $\begin{array}{c}\text { Уровень 1 } \\
\text { фактора 1 }\end{array}$ & $\mathrm{w}_{1}$ & $\mathrm{y}_{1}$ \\
\hline$\ldots$ & $\ldots$ & $\ldots$ & $\ldots$ & $\ldots$ & $\ldots$ \\
\hline $\begin{array}{c}\text { Уровень n } \\
\text { Фактора 1 }\end{array}$ & $\begin{array}{c}\text { Уровень } \mathrm{n} \\
\text { Фактора 2 }\end{array}$ & $\ldots$ & $\begin{array}{c}\text { Уровень } \mathrm{n} \\
\text { Фактора 1 }\end{array}$ & $\mathrm{w}_{1}$ & $\mathrm{y}_{1}$ \\
\hline
\end{tabular}

Как правило, при большом количестве факторов экспозиция (табл.1) в большинстве ячеек wi слишком мала, для того чтобы производить оценку поздно заявляемых убытков на основе вышеназванных моделей (дисперсия такой оценки будет неприемлемо велика с практической точки зрения).

Практический выход из этой ситуации - сгладить влияние отдельных факторов на размер поздно заявляемых убытков, произведя оценку на основе всей массы экспозиции (или отдельных ее крупных частей) и распределив величину неизвестных на дату расчета тарифа убытков по ячейкам табл.1 на базе экспозиции риска.

Очевидно при таком подходе (на примере автострахования), что вероятность заявления в будущем убытков по событиям прошлых лет от владельца автомобиля, используемого в такси, выше, чем вероятность заявления владельцем мотоцикла, однако при одинаковой экспозиции риска и тот, и другой получат 
одинаковые оценки убытков, которые будут заявлены в будущем. Все это способно несколько исказить тариф: занизить тариф для такси и завысить его для мотоцикла.

В части второй рассмотрен способ улучшения тарифа в данных обстоятельствах с применением инструментария обобщенных линейных моделей.

Часть II. Оцуенка резерва поздних убытков (IBNR) с применением обобщуенныхх линейных моделей

В качестве теоретической базы предлагаемого подхода взята простая модель оценки IBNR - модель независимых нормированных приращений убытков (МНП). Основные предпосылки этой модели ниже.

Рассмотрим треугольник развития убытков (табл.2).

Таблица 2

Треугольник развития убытков

\begin{tabular}{|c|c|c|c|c|c|}
\hline $\begin{array}{l}\text { Период собы- } \\
\text { тия (i) / разви- } \\
\text { тия (k) }\end{array}$ & 1 & 2 & $\ldots$ & I-1 & I \\
\hline 1 & $\mathrm{~S}_{11}$ & $\mathrm{~S}_{12}$ & $\ldots$ & $\mathrm{S}_{1 \mathrm{I}-1}$ & $\mathrm{~s}_{1 \mathrm{I}}$ \\
\hline 2 & $\mathrm{~S}_{21}$ & $\mathrm{~S}_{22}$ & $\ldots$ & $\mathrm{s}_{2, \mathrm{I}-1}$ & \\
\hline$\ldots$ & $\ldots$ & $\ldots$ & $\ldots$ & & \\
\hline $\mathrm{I}-1$ & $\mathrm{~S}_{\mathrm{I}-1,1}$ & $\mathrm{~S}_{\mathrm{I}-1,2}$ & & & \\
\hline I & $\mathrm{S}_{\mathrm{I} 1}$ & & & & \\
\hline
\end{tabular}

Символом $\mathrm{s}_{\mathrm{ik}}$ принято обозначать приращение убытков от событий периода i за период развития $\mathrm{k}$ (число или сумма заявлений в периоде либо число или сумма выплат). В качестве периода обычно используется год.

В рамках модели МНП все $s_{i k}$ предполагаются независимыми случайными величинами [2, с.205-206], и показатели $\mathrm{s}_{\mathrm{ik}} / \mathrm{w}_{\mathrm{i}}$ имеют одинаковое математичекое ожидание $\mathrm{m}_{\mathrm{k}}$ и дисперсию, отличающуюся только из-за объема риска (экспозиции риска) $\sigma_{k}^{2} / w_{i}$, где $\sigma^{2}{ }_{k}-$ общий для всех периодов событий параметр дисперсии.

Предлагаемая факторная детализация заключается в следующем.

Шаг 1.

Рассматриваем $\mathrm{s}_{\mathrm{ik}}$, как сумму убытков по уровням факторов, используемых для подсчета тарифа (табл.1). 


$$
s_{i k}=s_{i k 1}+\cdots+s_{i k n}
$$

где $s_{i k 1}, s_{i k n}-$ приращения убытков в к-м периоде развития, соответствующие пересечению уровней факторов в 1-й, n-й ячейках табл.1 соответственно.

Математическое ожидание нормированного приращения убытков в к-м периоде развития таким образом исходя из формулы (1) определяется формулой (2).

$$
E\left(\frac{s_{i k}}{w_{i}}\right)=E\left(\frac{s_{i k 1}}{w_{i 1}}\right) \frac{w_{i 1}}{w_{i}}+\cdots+E\left(\frac{s_{i k n}}{w_{i n}}\right) \frac{w_{i n}}{w_{i}},
$$

где $E$ - оператор математического ожидания/

Как видно из формулы (2) математическое ожидание нормированного приращения убытка в периоде к неодинаково для всех периодов событий по причине различной структуры $\frac{w_{i j}}{w_{i}}$ портфеля в каждом периоде события (на практике она не может быть одинаковой для всех периодов).

Шаг 2.

Далее предполагаем, как и в случае с МНП неизменность математического ожидания нормированного приращения убытка в ячейке табл.1 с номером ј от периода к периоду, так что справедливо (3).

$$
E\left(\frac{s_{i k j}}{w_{i j}}\right)=m_{k j}
$$

Как отмечалось выше при рассмотрении табл.1 из-за малого количества экспозиции в отдельных ячейках невозможно дать точную оценку показателям (3) подобно оценкам МНП, как предлагает Т.Мак [2, с.206]. Поэтому переходим к шагу 3.

\section{Шаг 3.}

Используем предположение об обобщенной линейной модели $m_{k j}$ от факторов.

Вектор математических ожиданий нормированных приращений убытков по отдельным ячейкам табл.1 определяем формулой (4).

$$
\overrightarrow{m_{k}}=\left(\begin{array}{c}
m_{k 1} \\
\ldots \\
m_{k n}
\end{array}\right)=\left(\begin{array}{c}
g^{-1}\left(\theta_{k 1}\right) \\
\ldots \\
g^{-1}\left(\theta_{k n}\right)
\end{array}\right) \text {, }
$$


где $g$ - каноническая функция связи [4, с.21] для рассматриваемого распределения нормированного приращения убытка,

$\theta_{k 1}, \theta_{k n}-$ канонические параметры распределения нормированного приращения убытка в і-й и n-й ячейках соответственно.

Таким образом, вектор канонических параметров исходя из известного инструментария обобщенных линейных моделей определяется формулой (5).

$$
\overrightarrow{\theta_{k}}=X_{k} \beta_{k}
$$

$X_{k}$ - матрица плана модели k-го года развития, отражающая принадлежность і-го наблюдения (строки табл.1) уровню фактора, за который отвечает соответствующий коэффициенту этого уровня столбец матрицы (из 0 или 1) для категориальных факторов, или значение уровня фактора для числовых.

$\beta_{k}-$ вектор коэффициентов модели за уровни категориальных факторов, a также коэффициентов за числовые факторы для модели k-го года развития.

Относительно параметра дисперсии выбранного распределения экспоненциального семейства предположение МНП сохраняется: $\sigma^{2}{ }_{k} / \mathrm{w}_{\mathrm{ij}}$, где $\sigma_{\mathrm{k}}^{2}-$ общий для всех периодов событий и уровней факторов параметр дисперсии. Однако в случае с ОЛМ дисперсия является уже функцией от параметра $\sigma^{2}{ }_{k} / \mathrm{w}_{\mathrm{ij}}$, ее равенство данному параметру действительно только для нормального распределения величин $\mathrm{s}_{\mathrm{ikj}} / \mathrm{w}_{\mathrm{ij}}$.

Оценка параметров обобщенных линейных моделей может производиться любыми доступными способами, имеющимися в распоряжении актуария на сегодняшний день в достаточном количестве.

Такая оценка параметров моделей $\beta_{k}$ для всех периодов развития, кроме первого, позволит заместить неучтенные поздние убытки оценками с применением данных моделей. Эти оценки будут лучше отражать факторную природу происхождения убытков по страховым событиям и позволят более точно построить единую тарифную многофакторную модель в последствии. Попытка учета качества периода события, как это предполагают модели цепной лестницы, Бюльмана-Штрауба или перекрестной параметризации также может быть предпринята добавлением фактора периода события в набор используемых факторов. 


\section{Bblвodbl}

В результате проведенной работы предложен метод оценки резерва поздних убытков (IBNR) на основе многофакторной модели, учитывающей факторную структуру тарифицируемого риска. Применение такого метода позволит актуарию более точно учесть влияние отдельных факторов на размер нетто-тарифа.

\section{Список литературы}

1. Бауэрс Н. Актуарная математика. Перев. с англ. / Н. Бауэрс [и др.]; под ред. В.К. Малиновского. - М.: Янус-К, 2001.

2. Мак Т. Математика рискового страхования. - М.: Олимп-Бизнес, 2005

3. Танюхин А.В. Актуарная оценка поздних убытков: модель цепной лестницы или обобщенная линейная модель нормированных приращений убытков, имеющих распределение Пуассона? // Интерактивная наука. - 2020. - №7(53). C. $87-91$.

4. Anderson D., Modlin C., Feldblum S. «A Practitioner's Guide to Generalized Linear Models»// Towers Watson-2014

Танюхин Алексей Владимирович - канд. экон. наук, актуарий, Саморегулируемая организация актуариев «Ассоциация профессиональных актуариев», Москва, Россия. 